\title{
Using expert patients to deliver teaching in general practice
}

\section{INTRODUCTION}

Traditionally the medical profession has used patients to tell their story and to demonstrate signs in a passive role; patients have also been used as standardised cases for tests of clinical competence. More recently, extensive use of feedback from patients has been sought on such matters as access and communication. This feedback is now ingrained in general practice remuneration through the Quality and Outcomes Framework ${ }^{1}$ and in the Royal College examination. The Postgraduate Medical Education and Training Board (PMETB) document Patients Role in Healthcare - The Future Relationship Between Patient and Doctor $^{2}$ promotes the use of patients as teachers:

'Patients and service users should be much more involved in the teaching, delivery, and assessment of postgraduate medical education and training. Patients/service users and carers have a tremendous bank of experience and knowledge and clearly have a role to play in the development of postgraduate curricula and assessment systems. ${ }^{\prime 2}$

The National Service Framework on Mental Health also specifies that service users should be involved in planning, providing, and evaluating education and training. ${ }^{3}$

There is an evidence base to suggest that in particular settings such as presentations, seminars, mentoring, and giving feedback on performance, patients can offer unique qualities. ${ }^{4}$ The Royal College of Paediatrics and Child Health completed a project which looked at involving young children ${ }^{5}$ and subsequently recommended that children should be involved in the teaching and assessment of paediatricians. The physiotherapy department in Southampton used expert patients and recommended these patients be well prepared and be familiar with the outcomes of the session,

but not to the extent that spontaneity is lost:

\begin{abstract}
'It is probably best to allow them free reign in recounting their experience and to encourage students to approach the session with an open mind." 6
\end{abstract}

Professor Kieran Sweeney, highlighting his journey after receiving the diagnosis of mesothelioma, ${ }^{7}$ also exemplifies the insight that patients can bring to trainees' learning.

While there has been some patient involvement in curricula development at an undergraduate level it has been slower to develop at the postgraduate stage. However for this to happen there first needs to be a cultural change that values patients' and service users' opinions as a valuable addition to academic and professional opinion. ${ }^{2}$ In addition, there needs to be the development of guidelines for patients preparing for the learning encounter and a framework for evaluating the encounter. ${ }^{9}$

Patient-led education with postgraduate trainees has not been used in a systematic way in the Northern Ireland Deanery before. This paper attempts to explore the perceptions of a group of GP ST3 trainees before and after participating in a patientled teaching session.

Guidance on strengthening personal and public involvement (PPI) in health and social care has been issued by the Safety, Quality and Standards directorate of Northern Ireland's Department of Health, Social Services, and Public Safety:

\section{'High quality PPI can really change things for people who use services, both in their experience of services and the quality and safety of care'. ${ }^{8}$}

\section{SETTING}

Northern Ireland Deanery has over 1.5 million patients and there are nearly 200 General Practice Specialty Trainees in the 3-year programme. Teaching is organised through a day release scheme facilitated by Programme Directors (PDs). This teaching is problem based and linked to the curriculum. There is the occasional use of guest speakers, usually local consultants, GPs with special interests or allied health professionals, at these day release sessions. Expert patients have not previously been used to facilitate the delivery of learning inputs.

\section{METHOD}

A practice meeting identified patients who were experienced teachers in their daily lives and who had encountered primary and secondary care through recent significant illness. Expert patient $P$ is a 51 -year-old head teacher and accomplished soloist well experienced in public performances. She has just completed a course of chemotherapy for colonic carcinoma. Expert patient $\mathrm{J}$ is a 54 -year-old Deputy Director of the Regional Teaching Training Unit with many years experience of training and group work. $\mathrm{He}$ is undergoing chemotherapy for prostatic cancer. Expert patient $B$ is a 60 -year-old retired teacher who taught for many years in a school in the practice area. $\mathrm{He}$ has marked anklylosing spondylitis and underwent a colectomy a few years ago for intractable ulcerative colitis.

The patients asked for guidance. This was kept to a minimum to enable them to organise the session in their own style. They were given 1 month to prepare a teaching plan with a presentation time of 1.5 hours. The aim of the session was for trainees to better understand the patients' perspective of their management so that they could modify their subsequent management of patients in a more perceptive way. The expert patients were asked to keep didactic elements of instruction to a minimum, to ensure trainee participation through group work and to promote discussion. They were instructed not to identify individual professional carers or providers in their delivery. It was made explicit that the exercise was to be more than just recounting their experience. They were also encouraged to be entirely frank 
and honest about their experiences: there was no need to present a favourable history but rather a fair and balanced viewpoint.

The trainees consisted of 11 female and five male white ST3s aged 25-30 years. Prior to the patient-led inputs they were facilitated, in a formal group discussion, to explore their learning hopes for the sessions, their concerns or apprehensions, and what information they needed to maximise their learning from these sessions. After the patient-led inputs, in a facilitated evaluative discussion, they were asked to explore how far their hopes had been met, how realistic their apprehensions were, what information they now realised they would have needed before the session, and how their practice might change as a result of these patient-led learning events.

\section{RESULTS}

\section{Pre-session discussion}

In the discussion prior to the patient-led training the following learning hopes, concerns, and needs were:

\section{Learning hopes.}

- To actually learn something of value from listening to these patients.

- The patient perspective: to broaden our horizons when communicating with patients; help us learn rather than be defensive; stimulate humility.

- To help us understand if the patients are negative about doctors or primary/ secondary care without feeling defensive.

- To reinforce the importance of allowing patients to ventilate, thereby reminding us of why we chose to become doctors in the first place.

\section{Concerns.}

- It will only be superficial, that is, not analytical; anecdotal; not knowledge/ evidence based; too much focus on feelings/emotions; too personal/ subjective; not necessarily honest/ objective.

- We don't need this; we've had this before at medical school; we have patients in our own families; we spend our day doing this anyway; listening to patients; this would be better for hospital doctors; we are already empathic and objective when reflecting on our interactions with patients.
- The patient will be a complainer; more likely to volunteer if had negative experience.

- We might feel threatened by expert patients.

- Inarticulate patients not qualified to teach; a patient who was a doctor might be a better teacher.

- Patient becoming overly emotional during the teaching session.

Needs.

- A clear goal(s) for the session.

- Clarity regarding how patient(s) are selected.

- If session was two-way patients could appreciate our perspective and the constraints within which we operate.

- Clarity around boundaries, that is, what can/cannot be asked of the patient; for example, end-of-life decisions.

\section{Post-session discussions}

This discussion group, after the expert teaching session, consisted of nine of the 16 previous trainees (four male and five female). The other seven were absent due to illness or annual leave. In this discussion the hopes fears and needs, articulated prior to the session were re-assessed.

Hope: to learn something of value.

The trainees' learning outcomes included:

- Left with a worry about what happens when you refer to hospital.

- It was a useful revision.

- Some people can accept negative events and put on a positive spin, without umbrage; the resilience of the patient.

- A single negative event can sour a memory.

- Its important that patients can contact their GP throughout their journey so they don't feel alone.

- Deficiencies are related to funding and time more than people or personality.

- Important to empathise, to consider the family life of the patient.

- Emotional intelligence is important, but patients don't just want good interpersonal skills they also want practitioners who can do the job in an effective hands-on way.

- Keep paying into BUPA!

- Once the patient is referred, the GP has very little control.

- Shocked at the poor communication between hospital and GP.

Hope: to better understand the patient's perspective.

The trainees' responses to this included:

- It was a reinforcement of previous awareness.

- It was nothing additional to what we already know from our practice patients and the members of our families who are also patients.

- It gave insight into psychosocial issues with which patients have to cope, including organising cover and time off work and battling with NHS bureaucracy.

- The use of selective amnesia as a coping strategy.

Hope: to re-inforce the importance of allowing patients to ventilate.

The trainees' responses included:

- It wasn't a catharsis - they had clarity and objectivity.

- As GPs we have no control over where our referrals go.

- Leaving with a feeling not a knowledge.

- Leaving depressed.

- They highlighted the absence of emotional intelligence in the health service.

Concerns: the session will be overly personal and subjective.

The trainees responses included:

- It was a patient's personal and subjective narrative about their journey but there were clear lessons.

- It rooted us in the reality that we are caring for human beings.

- We can't extrapolate this to the entire population.

- It was uncomfortable listening to system failures.

Concerns: we know this already.

Trainees' responses included:

- We all took valuable points.

- Many negative points were more applicable to secondary rather than primary care.

- GPs are already patient and empathycentred.

- Important for the hospital staff to listen to this. 
- Their experience didn't shock me - l've seen it myself.

- I was initially cynical, but it was good to be reminded.

- Patients articulated well; it really helped.

- Reminded me what I need to do regarding communication between doctors and between the doctor and patient.

- The patients didn't want to impose on the practice.

- GPs can learn about the interface between primary and secondary care.

- GPs already reflect a lot through videos and case-based discussion.

- Those that need to work with their emotional intelligence don't know it.

Concerns: the patients will be overly negative about their experiences or not representative of the patient population.

Trainees' responses included.

- These patients were chosen, well educated, and so skewed.

- They were non-confrontational but this is not the norm.

- They gave honest stories, based on thought and effort and well communicated.

- A negative experience can be presented reasonably, but not every patient can do this.

- These patients were well educated yet had difficulty understanding the system, so what chance do others have - it would be very distressing.

- It didn't degenerate into overly personal, meandering, highly subjective rambling.

- They depersonalised it and made it less uncomfortable; otherwise we would be squirming.

- Patients in this role need to communicate but not every patient could and this would negate the process as you need a spectrum.

Concerns: feeling threatened by the expert patient.

Trainees' responses included:

- Not a problem.

- Not used as a weapon.

- It wasn't the main focus.

Concerns: patient not able to teach.

Trainees' all agreed:

- The patients are or were teachers and therefore able to teach in an appropriate way.

- They were chosen and therefore articulate.

Concerns: patients will be overly emotional.

Trainees responses included:

- Their stories were personal but not told in an overly emotional way.

- They were clear and objective.

- We didn't ask questions that might have stimulated an emotional response.

Pre-session need: clear learning goals for the session.

Trainees' responses included:

- The learning outcomes gained will vary from trainee to trainee.

- Don't try to measure goals/outcomes.

- Best not to prepare; just to have an open mind.

Pre-session need: clarity around how patients selected.

Trainees unanimous response:

- Yes.

Pre-session need: a two-way participative session.

Trainees' responses included:

- Yes patients had distance.

- There were less gripes about the GP.

- They had an agenda.

- It wasn't really two-way but didn't need to be.

- We are not apologists for the NHS.

Pre-session need: clear, appropriate boundaries.

Trainees' responses included:

- We didn't feel it was appropriate to ask about their real fears.

- We didn't want to go too close to their feelings - it was still raw.

- We could have got more if we asked more, but we didn't want to cause harm and not a lot more to get out of it.

- Perhaps a paragraph away from tears at times.

- Not fair, appropriate, or reasonable in this group setting to go further, easier on a one-on-one.

Finally the trainees were asked how, if at all, their practice will change as a result of these patient-led inputs. All indicated that their practice would change and their responses included:

- Don't take things at face value, push further.

- Awareness of our own emotions and how it affects the relationship.

- When referring patients keep in contact.

- Don't have PSA without seeing the doctor.

- Keep a diary of serious issues.

- New cancer diagnoses to be flagged and contacted and allowed a 20-minute appointment to discuss.

- Allow patients to ventilate without interrupting.

- Reassure patients that the GP is available.

- Promote the GP role to shepherd patients thorough the system.

\section{DISCUSSION}

It has been suggested that for patients to play a role in postgraduate medical education there needs to be a cultural change which values service users' opinions and inputs. ${ }^{2}$ This view was borne out by this study as, in the pre-session discussions, the trainees' apprehensions outweighed their hopes for the session. They were quite sceptical about whether patients had any insights to offer them that might enhance their awareness. The general view was that patients lacked the medical knowledge base to be of use. This was reflected in comments like:

'What are their qualifications?'

'They will lecture you on things that are anecdotal rather than evidence-based.'

'I don't feel they should be called experts or educators.'

Moreover there was a suspicion about the motivation of patients who wished to undertake this role. They were likely to be people who had a negative experience and who wished to inflict their negativity on the trainees. This was expressed in comments such as:

'Patients complain ... they are more likely to volunteer if they have a negative experience'. 
An interesting variation on this was that the patients would not be able to cope with the emotion of the occasion and this would create embarrassment for the trainees.

Their lack of enthusiasm for what was being proposed was also evidenced by their belief that they had experienced patient inputs at undergraduate level and therefore this would be a repetition of what they already knew. This was articulated in statements like:

'We have had experience of this at medical school.'

A variation of this theme was the argument that GP trainees don't need this because:

'We spend all day listening to patients telling us their stories.'

Therefore, it was argued, these patients could spend their time more profitably talking to hospital doctors who were less skilled in empathic awareness than these trainees were.

It must be said however that some group members did express their hopes for the session in an enthusiastic way, best reflected perhaps in the comment:

'This should remind us of why we chose to be doctors in the first place'.

However this was a minority view. Therefore these comments and attitudes symbolise the culture that needs to be changed: the belief that only clinical experts can be of value to trainees and that they can't learn from the people who are the actual service user. It would be tempting to see this as rooted in over-confidence or lack of self-awareness but it might also be linked to insecurity as evidenced by the comment:

'I might feel threatened by a patient who was an expert in his or her condition'.

In the post-sessions evaluation there was an interesting reaction from the trainees. Most of their apprehensions had proved to be misconceived. While the patient inputs were personal and subjective this was now perceived to be of value because:
'It rooted us in the reality that we are caring for human beings'.

While the trainees initially felt this was needless repetition of what they already knew now:

\section{'It was a useful reminder of what I need to do while I am communicating with other doctors and with my patients'.}

Rather than being overly negative the patients who delivered the sessions were 'non-confrontational' with 'honest stories' who showed that 'a negative experience can be presented reasonably.' Finally when asked what, if anything, they might change in their practice as a result of the sessions, each member of the group, including those who had been most sceptical beforehand, indicated one thing they would change.

There was therefore a significant attitude change in the group after the patient-led sessions in that they were much more aware of the value of hearing patients in this context.

It is important not to overestimate this attitude change because there were enthusiastic voices expressing their hopes for the sessions before they took place. Equally there was still some cynicism around afterwards exemplified in the views that:

\section{'I would gain as much from patients in my own family.'}

'These were not normal patients because they were chosen and not every patient can present as reasonably as this'.

Moreover we must also remember that these trainees may not be a representative sample of GP trainees within the Northern Ireland Deanery.

\section{CONCLUSION}

Patient-led inputs into postgraduate medical education require a change of culture. This study suggests that this culture change can be achieved by facilitating patients with effective communication skills to actually take teaching sessions, as trainee cynicism can be overcome by the realisation that they can learn and change their practice as a result of patient-led inputs. Therefore it seems reasonable that such events should be organised, on a pilot basis, throughout the Deanery and that patients should be considered for involvement in developing assessments and the evaluation of medical education events. This study suggests that these proposals might, initially, be met with suspicion by some GPs but that this attitude might well change as a result of experiencing the enhancement patient involvement can bring.

\section{Fergus Donaghy, Oliver Boylan and Claire Loughrey}

\section{REFERENCES}

1. Department of Health. Quality and Outcomes Framework (QOF).

http://www.dh.gov.uk/en/Healthcare/Primarycare/Pri marycarecontracting/QOF/index.htm (accessed 18 Jan 2010).

2. The Postgraduate Medical Education and Training Board. Patients' role in healthcare - the future relationship between patient and doctor. http://www.pmetb.org.uk/fileadmin/user/Content_an d_Outcomes/Working_group_reports/Patients_Role_i n_Healthcare_working_group_report20080620_vl.pd $\mathrm{f}$ (accessed 18 Jan 2010).

3. Department of Health. National Service Framework for Mental Health: modern standards and service models. http://www.dh.gov.uk/en/Publicationsandstatistics/Pu blications/PublicationsPolicyAndGuidance/DH_40095 98 (accessed 13 Jan 2010).

4. Wykurz G. Developing the role of patients as teachers; literature review. BMJ 2002; 325: 818-821.

5. Royal College of Paediatrics and Child Health Coming out of the shadows: a strategy to promote participation of children and young people in $\mathrm{RCPCH}$ activity.

http://www.rcpch.ac.uk/search?search=Coming+out+ of +the+shadows $\% 3 a+a+$ strategy+to+promote+partic ipation+of+children+and+young + people + in + RCPC $\mathrm{H}+$ activity (accessed 18 Jan 2010).

6. Ottewill R, Demain S, Ellis-Hill C, et al. An expert patient-led approach to learning and teaching: the case of physiotherapy. Med Teach 2006; 28(4): e120-e126.

7. Sweeney K, Toy L, Cornwell J. A patients journey: mesothelioma. BMJ 2009 Aug 14;339:b2862. doi 10.1136/bmj.b2862.

http://www.bmj.com/cgi/content/abstract/339/aug14 1/b2862 (accessed 13 Jan 2010).

8. Department of Health Social Services and Public Safety. Guidance on strengthening personal and public involvement in health and social care. http://www.dhsspsni.gov.uk/userinvolvement.pdf (Accessed 13 Jan 2010).

9. Howe A, Anderson J. Involving patients in medical education. BMJ 2003; 327: 326-328.

DOI: 10.3399/bjgp10X483346 